\title{
Zur Hymenopteren-Fauna der Umgegend von Frankfurt a. M.
}

von

F. Jaennicke.

Nachfolgend gebe ich, unter der Voraussetzung, dafs sie für die Herren Hymenopterologen nicht ohne Interesse sein werde, eine Uebersicht der von mir, während der letzten drei Jahre in hiesiger Gegend gesammelten Hymenopteren (496 Arten). Wenn das Verzeichnifs auch selbstverständlich nicht die entferntesten Ansprüche an Vollständigkeit zu machen im Stande ist, so dürfle dasselbe doch jedenfalls alle gemeinen und wohl weitaus die meisten der hier häufiger vorkommenden Arten (mit Abrechnung der Microhymenopteren) enthalten, wie es andererseits auch eine nicht geringe $\mathrm{Zahl}$ seltenerer Species aufweist und so wenigstens einen allgemeinen Ueberblick der Hymenopterenfauna unserer Gegend zu geben geeignet ist.

Was die Bestimmung betrifft, so mufs ich erwähnen, dafs mir hierin die Herren Medicinalrath Dr. Reinhard in Dresden und Professor Dr. Schenk in Weilburg bereitwilligste Hülfe leisteten, ohne welche der gröfsere Theil der Sammlung wohl noch längere Zeit unbestimmt geblieben wäre. Bei dieser Gelegenbeit erlaube ich mir den Wunsch auszusprechen, es 'möchten sich einige Fachmänner der einheitlichen Bearbeitung eines, das ganze hymenopterologische Gebiet umfassenden, Handbuches zum Bestimmen, ähnlich wie die Handbücher von Redtenbacher und Schiner, unterziehen. Bei der grofsen Zerstreuung der Arbeiten auf diesem Gebiet dürfte ein solches Unternehmen jedenfalls ein höchst verdienstliches und der Sa. che förderliches sein.

Zum näheren Verständnifs des Verzeichnisses bemerke ich folgendes., Diejenigen Species, bei welchen ein bestimmter Fundori nicht angegeben ist, sind au den verschiedensten Orten der Umgebung Frankfurts, bei Spaziergängen u. s. w. gesammelt worden. 
Die erwähnte Gegend bei Rüsselsheim erinnert in ihrer Fauna vielfach an die der Mombacher Heide. Diejenigen, bei welchen das Geschlecht ohne weitere Zahlen angegeben ist, habe ich nur in dem betreffenden einen Exemplare gefangen. Diejenigen Arten endlich, welche einer näheren, Geschlecht oder Zahl betreffenden Angabe entbehren, habe ich mehrfach, jedoch meist nur in geringer Anzahl gesammelt, ohne genügende Anhaltspuncte zu einem Schlusse auf häufigeres Vorkommen u. s. w. zu haben.

\section{Anthophila.}

Apis mellifica L. (mit Uebergängen zu A. ligustica).

Bombus terrestris L. sehr gemein die + , nur $1 \delta^{\top}$ und 1 ㅎ.

var. lucorum L. sehr häufig im Taunus, besonders $\delta$. Mehrere $q$ auch bei Rüsselsheim und im Frankfurter Wald.

- hortorum L. nicht häufig.

- Latreillellus K. 2 9 Schwanheim.

- fragrans $\mathrm{Pz}$. aufdem Kolbenberg im Mai an Calluna vulgaris.

- lapidarius L. sehr gemein.

- pomorum Pz. $1 \sigma^{\top}$ und ఫ̧. Frankf. Wald.

- Rajellus K. 3 오 ebendaher.

- subterraneus L. var. soroënsis K. im Taunus nicht häufig.

- hypnorum L. scheint selten; 1 오 und $\Varangle$ bei Rüsselsheim.

- pratorum L. (nur ఫ̧) nicht selten.

- $\quad$ - var. subinterruptus K. nicht häufig.

- muscorum F. selten. Frankfurter Wald.

- agrorum F. gemein.

- sylvarum L. desgl. Schwanheim, Taunus.

Psithyrus rupestris F. $\delta$ Taunus und Schwanheim. campestris Pz. Schwanheim.

var. Leeana "K.

- Francisana K.

- Barbutellus K. nicht selten.

- vestalis Fourcr. desgl.

Anthophora retusa K. häufig.

- $\quad$ aestivalis $\mathrm{Pz}$. ㅇ.

- fulvitarsis Lep. .

- quatrimaculata F. $\delta$.

- furcata $\mathrm{Pz}$. ․ Hochheim.

Eucera longicornis $\mathrm{L}$. nicht häufig.

Rhophites quinquespinosus $8 \mathrm{p}$. 
Melecta punctata K. im Fṛühjahr im Botan. Garten an Aubrietia deltoides in Menge gesammelt.

Melecta luctuosa Scop. 오. Mombach.

Crocisa histrionica F. 오.

Epeolus variegatus L. 오 Kelsterbach.

Nomada succincta $\mathrm{Pz}$. nicht selten 오.

Marshamella K. 오.

- lineola Pz. ㅇ. Rüsselsheim.

- sexfasciata Pz.

- Jacobaeae Pz. ठ'. Rüsselsheim.

Solidaginis Pz. Rüsselsheim auf Thymus Serpyllum.

ruficornis L. häufig. ㅇ.

zonata $\mathrm{Pz}$. 오. Mombach.

flava $\mathrm{Pz}$. nicht häufig.

- var. signata Jur. 오.

Roberjeotiana Pz. Rüsselsheim im Aug. in Menge auf Thymus Serpyll.

- alboguttata H. S. ð Mombach.

- ferruginata K. ㅇ. Rüsselsheim.

- flavoguttata K. ㅇ. Hoechst.

- minuta F. ㅇ.

Xylocopa violacea F. Frühjahr und Oct. im Bot. Garten in Menge schwärmend.

Panurgus lobalus F. $\delta$.

Dufourea vulgaris Sch. ‥

Halictoides dentiventris Ny $\mathrm{l}$. $\sigma$. Wiesbaden.

Dasypoda hirtipes F. Rüsselsheim und Kelsterbach sehr häufig. Aug.

Melitla tricincta K. desgl.

haemorrhoidalis F. Kelsterbach.

Andrena Hattorfiana F. ㅇ․

Schrankella Nyl. ठ’.

- cingulata K. nicht selten.

- rubricata Sm. ఠ. Schwanheim.

- Rosae Pz.

- cineraria L.

- pilipes F. nicht selten.

- nitida K. desgl. Kelsterbach.

- Trimmerana K. 2 б. im März an Stachelbcerblüthe im

Frankf. Wald (Grastränke).

ovina K. 오.

- nigroaenea K. 
Andrena tibialis K. ㅇ.

fulva Schrank im Garten an Arabis alpina.

- albicans K. nicht selten.

- fulvago Chr. $\sigma^{\top}$.

- varians Rossi nicht selten.

- helvola L. ㅇ. Frankfurter Wald.

- clypearis Nyl. ․ Taunus.

- Gwynana K. nicht selten.

- bicolor F. 3 ㅇ auf Arabis alpina im Garten.

- fulvicrus K. nicht häufig.

- fasciata Wesm. ㅇ auf Arabis alpina im Garten.

- Listerella K. . Taunus.

- decipiens Schenk 우. Rüsselsheim.

- albicrus K. April Mombach nicht selten.

- Wiekella K. 오. Rüsselsheim.

- fuscata K.

- convexiuscula K. Rüsselsheim.

- Afzeliella K. $\sigma^{\top}$. Frankfurter Wald.

- Lewinella K. Q. Taunus.

- cyanescens Nyl. 오. Frankf. Wald.

- proxima K. $ठ$. Taunus.

- parvula K. häufig.

Hylaeus Scabiosae Ill. 오 Frankf. Wald.

- sexcinctus F. Rüsselsheim nicht selten.

- laevigatus K. Wiesbaden.

- rufocinctus Sichel \& Taunus.

- sexnotatus K. Rüsselsheim.

- leucozonius K. nicht selten.

- quadricinctus F. Frankf. Wald.

- rubicundus Chr. Schwanheim nicht selten.

- maculatus Sm. nicht selten.

- cylindricus F. Frankf. Wald.

- fulvocinctus K. überall gemein.

- - var. abdominalis K. im Frankf. Wald n. s.

- albipes F. 우 Taunus.

- prasinus Sm. Schwanheim.

- obovatus K. Frankf. Wald.

- pauxillus Schenk ㅇ.

- minutus K. ㅇ. Frankf. Wald.

- parvulus Schenk Taunus.

- nitidiusculus $\mathrm{K}$. 우. ebend. 
Hylaeus flavipes F. nicht häufig. minutulus Schenk $\sigma^{\prime}$.

Smeathmannellus K. ㅇ.

morio F. Taunus.

- leucopus K. ㅇ․ ebend.

Colletes fodiens K. Rüsselsheim und Kelsterbach 1). s. Aug.

Daviesana K. nicht selten.

cunicularia L. April Mombach.

Sphecodes ,fuscipennis Germ. nicht h. Frankf. Wald.

gibbus L. nicht selten. Rüsselsheim.

rufescens F ourcr. Rüsselsheim.

subquadratus Sm. nicht selten. Taunus.

ephippium L. n. s. Taunus und Rüsselsheim.

Prosopis variegala F. ㅇ․ Kelsterbach.

- confusa Nyl. $\sigma^{\circ}$ im Garten auf Arabis alpina und Alyssum serpyllifolium.

- armillata Nyl. ㅇ․ ebendas.

- pictipes Nyl. 우 ebend.

- communis $\mathrm{Nyl}$. ebendas. nicht selten.

- propinqua Nyl. ㅇ. ebendas.

Megachile maritima K. $\sigma^{7}$. Kelsterbach.

- fasciata Sm. ㅇ. Frankf. Wald.

- circumcincta K. Schwanheim.

- centuncularis L. ㅇ. Kelsterbach.

- $\quad$ argentata F. Kelsterbach.

Trachusa Serralulae Pz. ebendas.

Osmia cornuta Latr. gemein.

- bicornis L. gemein. Ich fand auch Zellen mit Puppen im Sept. bei einem Schuhmacher in der Kehle eines Schuhleistens, welcher längere Zeit auf dem Speicher gelegen hatte und gingen die Puppen im Oct. aus.

- aurulenta Pz. 오. Rüsselsheim.

- fulviventris $\mathrm{Pz}$. nicht selten.

- aenea L. Taunus.

- adunca Ltr. nicht selten.

- Spinolae Ltr. Hochheim.

Anthidium manicatum L. häufig im Aug. auf den Sandhügeln bei der Kelsterbacher Windmühle.

- oblongatum Ltr. ebenda jedoch weniger häufig.

- $\quad$ punctalum Ltr. desgl.

Heriades nigricornis $\mathrm{Nyl}$. nicht selten.

Berl. Entomol. Zeitschr. XI. 
Heriades Campanularum K. 우.

Stelis aterrima Pz. Rüsselsheim.

Coelioxys conica L. Schwanheim.

- punctala Lep. Kelsterbach.

\section{Vespidae.}

Vespa Crabro L. einmal in grofser Zahl an einer Esche schwärmend gefunnden.

- holsatica F. ㅇ. Schwanheim.

- vulgaris L. häufig.

- germanica F. sehr gemein.

- mufa L. nicht häufig.

Polistes diadema Latr. einmal an einem Nest bei Königsstein in grofser Zahl gefangen; sonst nicht mehr gesehen.

- gallica L. gemein.

Eumenes pomiformis Rossi. Rüsselsheim.

Discoelius zonatus Pz. ㅇ. Kelsterbach.

Symmorphus crassicornis Pz. ㅇ. Königsbrunnen.

- elegans H. S.

- sinuatus F. nur 오.

Ancistrocerus renimacula Lep. Frankfurter Wald.

parietum L. gemein.

- $\quad$ oviventris Wesm.

- Antilope Pz. 오.

Leionolus simplex F. ㅇ․

- nigripes Pz. $\sigma^{7}$.

- exilis H. S. 우. Taunus.

Hoplopus spinipes H. S.

III. Sphegidae.

Crabro striatus H. S. am Königsbrunnen, nicht sellen.

Solenius sexcinctus v. d. L. ㅇ. Frankf. Wald.

- cephalotes H. S. Frankf. Wald. Königsbrunnen.

- lapidarius Pz. ㅇ. Taunus.

Eetemnius guttatus v. d. L. $\sigma^{7}$. Mombach.

- vagus L. ㅇ. Kelsterbach.

Thyreopus cribrarius L. desgl.

patellatus v. d. L. ㅇ. Frankf. Wald. Königsbrunnen.

Ceratocolus vexillatus Pz. Frankf. Unterwald.

Crossocerus cetratus $\mathrm{Sh}$. Rüsselsheim.

leucostoma L. ㅇ. Frankf. Wald. Königsbrunnen. 
Blepharipus signatus H. S. $\sigma$. Rüsselsheim.

Lindenius albilabris F. Rüsselsheim nicht selten.

Oxybelus dissectus Dhlb. ㅇ․ Kelsterbach.

Trypoxylon figulus L. $\sigma^{\circ}$.

Pemphredon lugubris $\mathrm{L}$ tr. nicht selten.

Cemonus rugifer Dhlb. $\delta$. Frankf. Wald. Königsbrunnen.

Passaloecus gracilis Curt. 오. im Garten.

Philanthus triangulum F. bei der Kelsterbacher Windmühle an Eryngium gemein.

Cerceris variabilis Dhlb. einzelne Stücke von Rüsselsheim, Taunus und Frankf. Wald.

- $\quad$ arenaria L. Rüsselsheim nicht selten. 1 ㅇ Taunus.

- nasuta Kl. $\sigma^{\top}$. Kelsterbach.

- labiala F. nicht häufig.

- interrupta Pz. Rüsselsheim gemein auf Thymus Serpyllum.

Nysson maculatus v. d. L. ㅇ. Mombach.

Gorytes campestris L. Mombach.

Hoplisus laticinctus Le p. $\sigma^{\top}$. Rüsselsheim.

Bembex rostrata L. Mombach nicht selten. - 1 \& Rüsselsheim.

Mellinus arvensis L. ㅇ. Rüsselsheim.

sabulosus F. ㅇ. desgl.

Tachytes pectinipes L. April Mombach nicht selten.

Miscophus bicolor v. d. L. Kelsterbach nicht selten.

Dinetus pictus F. 오, Rüsselsheim.

Anmophila sabulosa L. gemein.

Miscus campestris Ltr. Rüsselsheim.

Psammophila viatica L. Mombach und Rüsselsheim.

affinis Kirby nicht selten Frankf. Wald.

Mimesa equestris F. ㅇ․ Rüsselsheim.

Psen atratus Pz. Kelsterbach häufig.

\section{Pompilidae.}

Pompilus cinctellus v. d. L. Rüsselsheim nicht selten.

niger F. Rüsselsheim.

rufipes L. ㅇ. Kelsterbach.

neglectus Wesm. desgl.

anceps $\mathrm{W}$ esm. desgl.

viaticus $\mathrm{L}$. überall gemein.

Priocnemis bipunctatus F. ㅇ. Kelsterbach.

fuscus F. nicht selten im Taunus. maculipennis Schenk 오. Kelsterbach. 
Priocnemis obtusiventris Schiö dt. 오. Frankf. Wald. notatus v. d. L. \&. Rüsselsheim.

Agenia punctum F. 우.

Pogonius intermedius Dhlb. ㅇ. Taunus. variegatus $\mathrm{L}$. 우. Rüsselsheim.

Ceropales maculata F. ㅇ․ nicht selten.

Ampulex europaea Gir. ㅇ. im Garten.

$$
\text { V. Sapygidae. }
$$

Sapyga punctata Kl. 우. Wiesbaden.

\section{Scoliadae.}

Scolia quadripunctata F. nicht selten bei Rüsselsheim und Kelsterbach auf Thymus Serpyllum und Eryngium.

Tiphia femorata F. Mombach und Rüsselsheim nicht selten.

- minuta v. d. L. ㅇ․ Kelsterbach.

- ruficornis Kl. $\sigma^{\top}$. Rüsselsheim.

VII. Mutillidae.

Mutilla europaea L. Taunus.

- maura F. ㅇ. ebendas.

- rufipes Ltr. ㅇ. Kelsterbach.

\section{Chrysidae.}

Chrysis succincta Msm. $\sigma$. Frankf. Wald.

- bidentata F. Taunus.

- indigotea Duf. ㅇ.

- ignita L. nicht selten, besonders bei Kelsterbach.

- - var. impressa Schenk. Kelsterbach.

- fulgida L. Schwanheim.

Hedychrum coriaceum Dhlb.

- $\quad$ ardens Ltr. ㅇ․ Kelsterbach.

- rutilans Dhlb. ebendas. häufig.

- regium Wesm. bei Kelsterbach und Rüsselsheim sehr gem. Nur wenige von Frankf.

Holopyga ovata $\mathrm{Dhlb}$.

Elampus auratus Wesm. ㅇ. Frankf. Wald.

- violaceus Scop. ㅇ․

- pusillus Wesm. nicht selten. 
IX. Formicidae.

Camponotus ligniperda Ltr. Taunns gemein. herculaneus L. Taunus nur 오.

Tapinoma erraticum Ltr.

Formica sanguinea Ltr. Frankf. Wald nicht selten.

- truncicola Nyl. ‥ Kelsterbach.

- rufa L. überall, jedoch nur einzeln.

- congerens Nyl. nicht selten Taunus.

- cunicularia Ltr. Schwanheim.

- fusca চ্ৰ. Kelsterbach.

Lasius fuliginosus L tr. gemein.

- niger L. Rüsselsheim nicht selten.

- alienus Först. Frankf. Wald häufig.

- brunneus Ltr. Frankf. Wald nicht selten.

- flavus F. Kelsterbach häufig.

- umbraticus Nyl. Frankf. Wald häufig.

Leptothorax acervorum F. Frankf. Wald.

Nylanderi Först. Taunus häufig.

Tetramorium caespitum L.

Myrmica rubida Ltr. ㅇ. Frankf. Wald.

- laevinodis Nyl. nicht selten.

- ruginodis Nyl. Taunus gemein.

- scabrinodis Nyl. Rüsselsheim gemein.

Diplorhoptrum fugax Ltr. Taunus.

\section{Ichneumonidae.}

Chasmodes lugens Grv. ${ }^{7}$.

Ichneumon extensorius Grv. nicht selten.

ferreus Grv. $\sigma^{7}$.

- comitator Grv. đo. Schwanheim.

- confusorius Grv. nicht selten.

- luctatorius Grv. Rüsselsheim.

- balteatus Wesm. ㅇ․ Frankf. Wald.

- $\quad$ stramentorius Grv. ㅇ․ Taunus.

- $\quad$ sarcitorius Grv.

- saturatorius Grv. 우. Mombach.

- annullator Grv. ㅇ.

- fabricator Grv. ฮ. Schwanheim.

- pallipes Grv. Taunus.

- pistorius Grv. 
Ichneumon lepidus Grv. Mombach.

digrammus Grv. ebendas.

Hoplismenus perniciosus Grv.

Amblyteles fasciatorius Grv. Taunus nicht selten.

- amatorius Grv. 우.

- oratorius $\mathrm{F}$.

- infractorius Pz. Mombach.

- litigiosus Wesm. Taunus.

- vadalorius III.

- unigultatus Grv. Rüsselsheim.

- castigator L. nicht selten.

- repentinus Grv. Schwanheim.

- Gravenhorstii Wesm. nicht selten auch Mombach.

- monitorius Pz. Schwanheim.

Trogus lutorius Grv.

- alboguttatus Grv.

Exochilum circumflexum L. ㅇ. Frankf. Wald.

Alomya ovator F. Rüsselsheim nicht selten.

Phygadeuon nycthemerus Grv.

- $\quad$ Reinhardii ơ. n. sp.

- $\quad$ gracile n. sp. 오.

- sp. dubia.

Cryptus peregrinator $\mathrm{Gr}$. nicht selten.

- opacus Gr. Schwanheim.

- adustus Gr. Kelsterbach.

- vidualorius F. Mombach.

IIemiteles areator Grv. Taunus.

- completus Ratzb.

- sp. dubia.

Pezomachus transfugus F. 오.

Rhyssa persuasoria L. 오

- approximalor F.

Thalessa obliterata Grv. Taunus.

Ephialtes manifestator Grv, häufig.

- carbonarius Chr.

- mediator F. ㅇ․

- rex ㅇ. Kelsterbach.

- sp. dubia.

Pimpla instigator F. 우. Rüsselsheim.

- turrionellae F. 오.

Glypta haesitator Grv. ㅇ. Kelsterbach. 
Glypta longicauda Grv. đ઼, Taunus.

mensurator Grv. \&. Rüsselsheim.

Lissonota conflagrata Grv.

- variabilis $\mathbf{H g r}$. 오.

Acoenites arator Rossi . . Kelsterbach.

Xylonomus filiformis Grv. ఫૅ. Frankf. Wald.

- irrigator F. 우 Taunus.

- aler Grv. đ. Schwanhein.

- gracilicornis Gr. 오.

Xorides dentipes Grv.

$$
\text { albitarsis Grv. 오. }
$$

Odontomerus dentipes Gmel. Taunus nicht selten.

Metopius migratorius Grv. $0^{7}$. Rüsselsheim.

Exochus mansuetorius Grv.

Colpotrochia elegantula Grv.

Catoglyptus Schenkii v. sp. ฮొ.

Tryphon elongatus F.

- incestus Hgr.

- vulgaris Hgr. Taunus.

- ephippium Hgr. Mombach und Rüsselsheim.

Ophion merdarius Grv. ơ. Frankf. Wald.

ramidulus $\mathrm{L}$. 오.

- obscurus Grv. häufig Mombach.

- luteus L. häufig.

- sp. dubia.

Paniscus glaucopterus Grv. häufig.

- testaceus Grv. Taunus, Kelsterbach.

Campoplex mixtus Grv.

- $\quad$ sp. dubia Taunus.

- - - Mombach.

Mesochorus sp. dubia.

Ischiogonus erythrogaster. W sm. 오.

Cteniscus flavomaculalus G rv.

Exetastes fornicator F. ఠ. Taunus.

Banchus pictus F. Ō. ebendas.

falcator $\mathbf{F}$. Kelsterbach nicht selten.

XI. Braconidae.

Microgaster glomerata L. häufig.

sp. dubia. 
Meteorus rubens Ns. ‥ Mombach.

- albitarsis $\mathrm{Curt}$. ㅇ. ebendas.

Helcon tardator Ns.

- aequator Ns. ㅇ․

- rusputor Ns. häufig.

- annullicornis Ns. 오

Earinus nitidulus Ns. Mombach.

Chelonus inanitus F.

Vipio terrefactor Vill.

Bracon flavator Ns. ㅇ. Frankf. Wald.

Coeliodes scolyticida W sm.

Dendrosoter protuberans Ns.

Spathius clavatus Ns. ơ. Taunus.

Alysia cephalotes $\mathrm{Hal}$.

XII. Evaniadae.

Foenus jaculator F. 오.

- affectator Ns. ㅇ.

Brachygaster minuta F.

XIII. Chalcidiae.

Smicra clavipes F.

Monodontomerus obsoletus Ns. Mombach.

Cheiropachys quadrum F. Kelsterbach.

Torymus admirabilis Först. 오.

Callimome sp. dubia.

Chalcis femorata Dhlb. Rüsselsheim.

Eurytoma sp. dubia.

Embolemus Rudddii W stw. \&? Kelsterbach.

XIV. Cynipidae.

Synergus sp. dubia.

Cynips tincloria L. Taunus.

XV. Tenthredinidae.

Cimbex variabilis $\mathrm{K}$. gemein.

Trichiosoma Betulae Zadd. Frankf. Wald.

Vitellinae L. ㅇ. Taunus.

- lucorum F. ebendas.

Clavellaria Amerinae F. ‥ Schwanheim. 
Abia sericea L.

Hylotoma Berberidis Sch r. Јૅ. Mombach.

ustulata L. Taunus und Rüsselsheim.

segmentaria $\mathrm{Pz}$. + .

coerulescens $\mathbf{F}$.

vulgaris K. Rüsselsheim.

enodis L. Rüsselsheim.

Rosarum F. sehr gemein.

mediata F. $\widetilde{c}$. Taunus.

Athalia spinarum F. Schwanheim gemein.

Rosae L.

Allantus marginellus Pz. Taunus.

zonula K. Schwanheim nicht selten.

tricinctus F. Frankf. Wald. nicht selten.

bicincta $\mathrm{L}$.

Scrophulariae L. 오. Rüsselsheim.

Macrophya neglecta Kl.

12-punctata L. Taunus nicht selten.

rustica L. ㅇ. ebendas.

Pachyprotasis Rapae I. nicht selten.

Tenthredo atra L. nicht selten.

- $\quad$ instabilis Kl. sehr gemein.

- lateralis Kl. $\sigma$. Frankf. Wald.

- punctulata Kl. häufig.

- obsoleta Pz.

- zonata Pz. 오. Taunus.

- flavicornis F. Taunus.

- viridis $\mathrm{L}$. 우.

Strongylogaster cingulata F. ㅇ․ Mombach.

Blennocampa ephippium Pz. Königsbrunnen.

- hyalina K. $\sigma^{\top}$. Taunus.

- fuliginosa Schr. ㅇ. Rüsselsheim.

Selandria straminipes K. Schwanheim.

serva F. Frankf. Wald.

Hoplocampa ferruginea F. ‥ Mombach. ovata F. ㅇ. Mombach.

Dineura rufa K. 우.

sp. dubia.

Emphytus melanarius K. $ఠ$.

Dolerus anticus $\mathrm{Kl}$.

haematodes Schr. ㅇ. Königsbrunnen. 
Dolerus gonager F. häufig.

carinatus Scholz ㅇ. Taunus.

vestigialis KI. Schwanheim.

niger K $\mathrm{l}$. Taunus.

anthracinus $\mathrm{K}$. ㅇ․ 'Taunus.

Nemalus Myosotidis F. häufig.

citreus $\mathrm{Z}$ add. 오.

poecilonotus $\mathrm{Zadd}$. 오. Taunus.

Cryptocampus angustus $\mathrm{Htg}$.

Lophyrus nemorum Kl. ㅇ․ Rüssselsheim.

virens $\mathrm{Kl}$. Mombach.

rufus KI. $\sigma$. Taunus.

Pini L. O־.

Lyda erythrocephala L. ㅇ․ Mombach.

- pratensis F. o. Frankf. Wald.

- reliculata L. ㅇ. ebendas.

- Klugii Htg. ${ }^{\top}$. Taunus.

- sylvatica L. Taunus und Mombach.

- arvensis $\mathrm{Pz}$. 우.

Cephus troglodytes $\mathrm{I}$.

- pygmaeus L. ơ. Frankf. Wald.

- spinipes Kl. nicht selten.

- floralis Kl.

- Satyrus Pz.

Oryssus vespertilio F. Taunus.

Xiphydria annulata Jur. Frankf. Wald.

Sirex gigas L. Taunus.

- juvencus L. 우. ebendas.

- spectrum L. ㅇ. ebendas.

Beschreibungen der aufgezählten neuen Arten.

Phygadeuon Reinhardii $\overbrace{}^{\star}$. n. sp.: Niger, abdomine nigroviolaceo; pedibus rufis; coxis trochanteribusque nigris, nitidis. - Long. 14 mill. - Patria: Germania.

Schwarz. Taster schwarz, Kopf und Gesicht grob punktirt und dicht, schwarz, etwas zottig behaart. Oberlippe gelbbraun. Fühler verdickt, borstenförmig von 10 Mill. Länge. Thorax schwarz, grob und körnig punktirt und ziemlich dicht, schwarzbraun behaart. Hinterrücken unvollständig gefeldert. Hinterleib langgestielt, stark gebogen, schwach glänzend, schwarz mit violettem Schimmer; in die Quere stark aber sehr fein nadelrissig und mit äufserst feinen weifslichen Härchen besetzt. Die Seiten punktirt. Beine roth; 
an den Hinterbeinen die Spitzenhüften der Schienen und die Tarsen schwarz. Flügel bräunlich mit kleinem schwarzem Randmal.

Fundort: Kelsterbach bei Frankfurt a. M.

Phygadeuon gracile n. sp. ‥: Niger; pedibus rufis, femoribus nigris; antennis albo annullatis; coxis, trochanteribusque nigris, apice rufis. - Long. 7 mill. - Patria: Germania.

Schwarz; das Endglied der Kiefertaster roth, Fühler fadenförmig, schwarz, das 8te bis 11te Glied gelblichweifs; die weifsen Glieder aufsen an der Spitze mit schwärzlichem Fleck. Kopf und Thorax dicht punktirt und auf der Oberseite dicht mit sehr kurzer brauner Behaarung bedeckt. Felderung des stark gekörnten Hinterrückens unregelmäfsig. Brustseiten stark glänzend. Hinterleib länglich eiförnig; dunkel schwarzbraun, glänzend. Das erste Segment mit starken Längsrunzeln; das zweite an der Basis fein der Länge nach gerunzelt und am umgeschlagenen Seitenrand schmal braungelb gerandet. Die Endsegmente mit gelben Hinterrändern. Stachel kurz. Schenkel schwarz. Schienen und Tarsen roth; die Spitze der Hinterschienen schwarzbraun. Hüften und Schenkelringe schwarz, an der Spitze roth. - Fundort: Kelsterbach.

Catoglyptus Schenkii n. sp. ठَ.: Niger; hypostomate flavo; antennis luteis, supra nigricantibus. Abdomine nitido, segmentis 2 et 3 margine postico albido marginatis. - Long. 15 mill. - Patria: Germania.

Stirne, Hinterkopf und Backen schwarz, weilläufig punktirt. Untergesicht hell citrongelb, glänzend. Taster röthlichgelb, die Wurzelglieder schwarz mit gelber Spitze. Wurzelglied der Fühler schwarz, auf der Unterseite mit weifslichgelbem Längsfleck. Fühler röthlichgelb, auf der Oberseite schwarzbraun. Thorax und Hinterrücken schwarz glänzend, mit kurzer, sehr feiner, weifslicher Behaarung dicht bedeckt und nicht sehr fein punktirt. Hinter dem Schildchen ein schmaler, weifsgelblicher Querfleck. Hinterrücken mit starken Leisten. Die inneren Felder gerunzelt. Hinterleib glänzend schwarz und fein punktirt, das zweite und dritte Segment mit breitem wachsweifsem Hinterrand. Hüften schwarz; die der Vorderbeine unten mit blafsgelbem Fleck. Schenkelringe gelb, oben schwarz; die der Hinterbeine schwarz mit gelbem Seitenfleck. Schenkel und Schienen der Vorder- und Mittelbeine unten und an den Knieen gelb, oben schwarz; die der Hinterbeine schwarz, an der Basis schmal gelb. Alle Tarsen gelblichweifs; Metatarsus der Mittelbeine oben schwarz; der der Hinterbeine mit ganz schwarzer Wurzelhälfte. Flügel gelbbräunlich, mit schwärzlich gerandeter Spitze und schwarzem Randmal. Spiegelzelle fehlt. - Fundort: Taunus. 


\section{$2 \mathrm{BHL}$ Biodiversity Heritage Library}

Jaennicke, F. 1867. "Zur Hymenopteren-Fauna der Umgegend von Frankfurt a. M." Berliner entomologische Zeitschrift / herausgegeben von dem Entomologischen Vereine in Berlin 11, 141-155.

https://doi.org/10.1002/mmnd.18670110112.

View This Item Online: $\underline{\text { https://www.biodiversitylibrary.org/item/36408 }}$

DOI: $\underline{\text { https://doi.org/10.1002/mmnd.18670110112 }}$

Permalink: https://www.biodiversitylibrary.org/partpdf/28554

\section{Holding Institution}

Smithsonian Libraries

\section{Sponsored by}

Smithsonian

\section{Copyright \& Reuse}

Copyright Status: Public domain. The BHL considers that this work is no longer under copyright protection.

This document was created from content at the Biodiversity Heritage Library, the world's largest open access digital library for biodiversity literature and archives. Visit BHL at https://www.biodiversitylibrary.org. 